\title{
THE SURGICAL MANAGEMENT OF CONGENITAL VERTICAL TALUS
}

\author{
Christopher L. Colton, London, England
}

From the Orthopaedic Department, Hospital for Sick Children, London

Congenital vertical talus is an uncommon condition which results in a severe, rigid, flat-foot deformity. It is distinguished from other types of pes plano-valgus by a set of clearly defined, diagnostic criteria which, if rigidly applied, result in the recognition of this abnormality only rarely. These criteria have been described by Lloyd-Roberts and Spence (1958) and are summarised below.

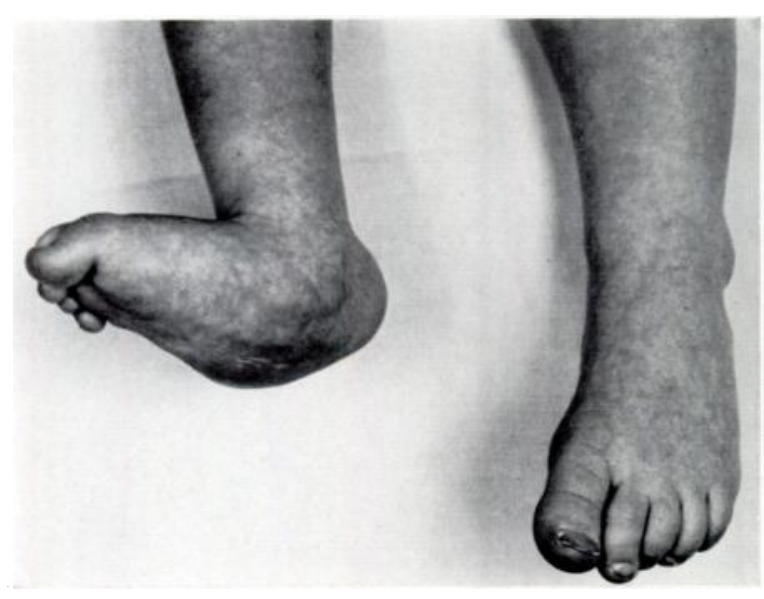

Fig. 1

The appearance of the typical deformity of congenital vertical talus.
FIG. 2

Plantar view of a foot with congenital vertical talus deformity showing how the rigid convexity of the sole fails to conform to the surface upon which it is standing.

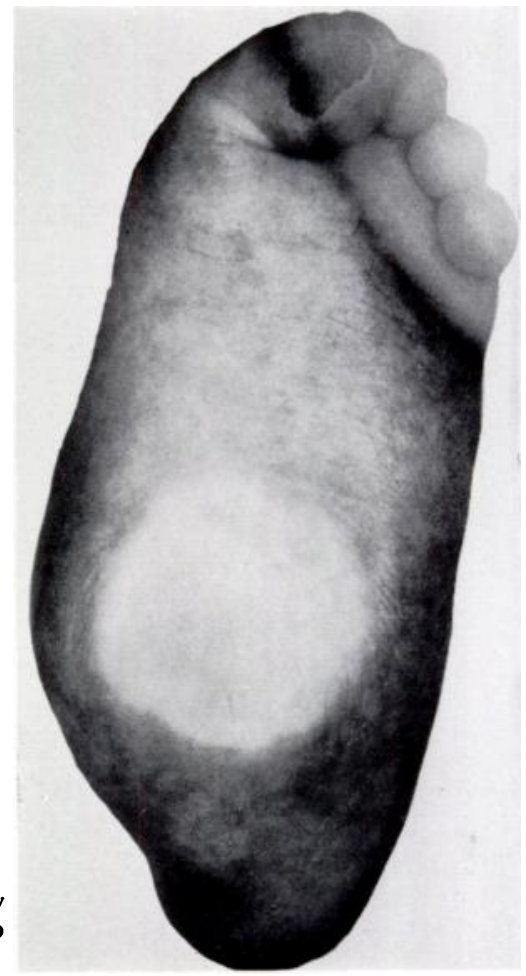

Typically, congenital vertical talus presents as a valgus posture of the foot with equinus of the heel, and abduction and dorsiflexion of the forefoot. This combination produces a "rocker-bottom" deformity (Fig. 1) with a concave lateral border to the foot. The head of the talus forms a prominence in the middle of the convex, medial border of the foot and a sulcus is present across the front of the ankle joint and the lateral malleolus. Above all, the deformed foot is rigid at the subtalar joint and on weight-bearing the lack of moulding of the foot to the ground is a prominent feature (Fig. 2). It is this rigidity of the foot in true congenital vertical talus which is responsible for its notorious lack of response to manipulative treatment (Lloyd-Roberts and Spence 1958; Herndon and Heyman 1963; Eyre-Brook 1967). Congenital vertical talus probably represents the severest deformity in a spectrum of abnormality which includes all forms of pes plano-valgus.

Radiologically the convex sole is easily recognisable and the long axis of the talus is seen to lie vertically in line with the tibia. The calcaneus is in equinus and after a few months of life it becomes narrowed and beak-shaped anteriorly. Once weight-bearing is assumed, a 
characteristic "hour-glass" appearance of the talus develops, probably from notching of the talar neck by the dorsally displaced navicular bone. The talar neck also appears to elongate during the first few years of life.
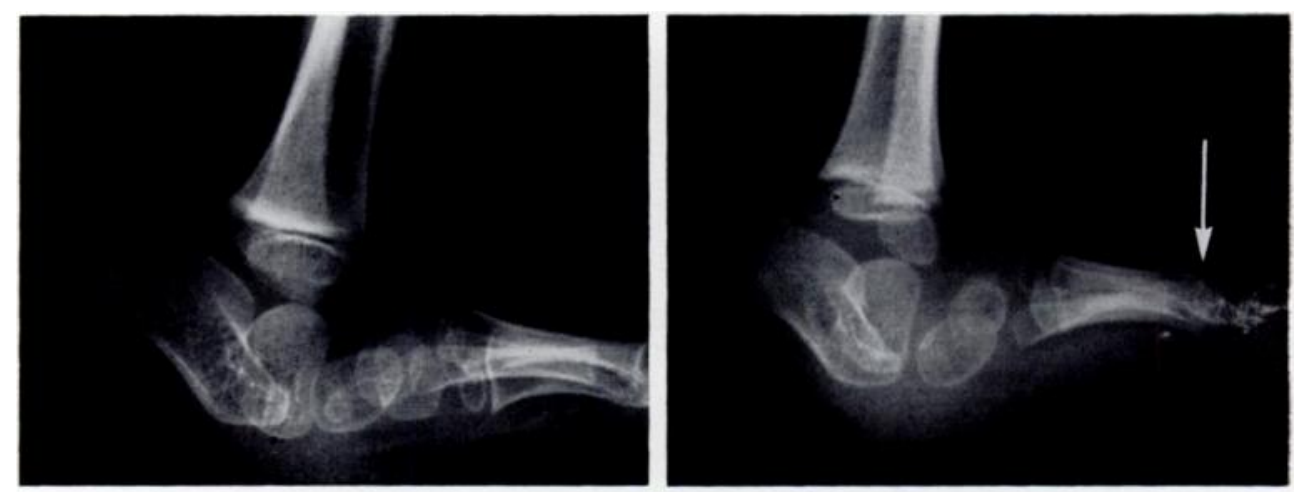

FiG. 3

Lateral radiographs of a foot with congenital vertical talus deformity taken in dorsiflexion and plantarflexion, showing the constant vertical orientation of the talus.
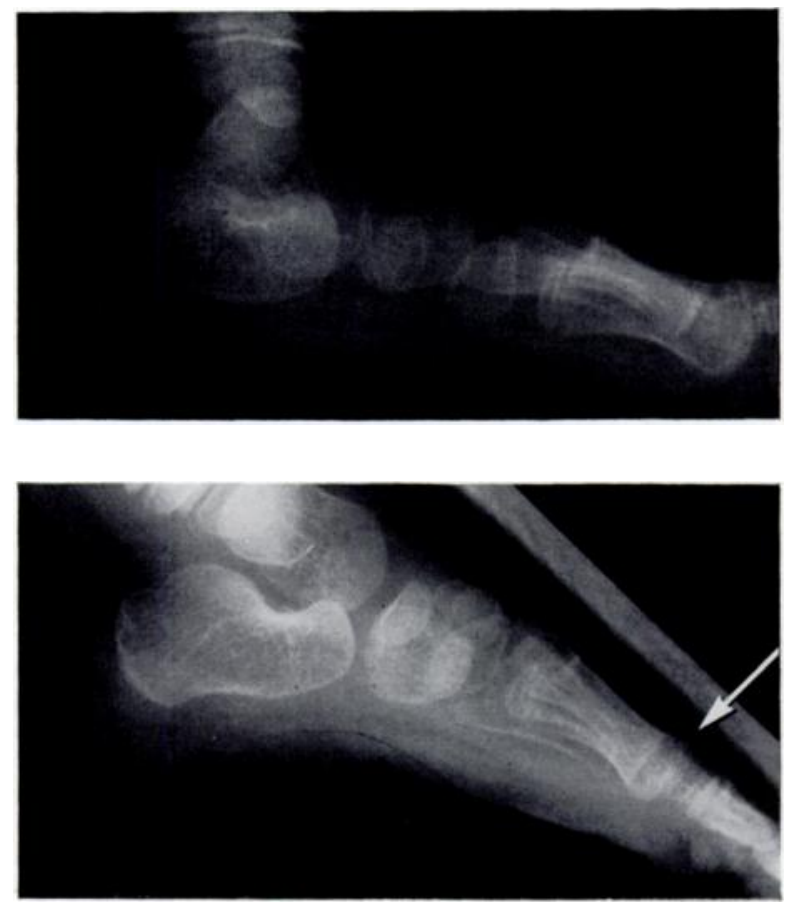

FiG. 4

Lateral radiographs in dorsiflexion and plantar-flexion of a foot with a mobile convex valgus deformity. Note the restoration of normal tarsal alignment in plantar-flexion.

The radiological features of congenital vertical talus have been described in detail by Haveson (1959). Eyre-Brook (1967) pointed out that in true congenital vertical talus the rigidity was radiologically demonstrated by the constant, abnormal relationship of the talus to the calcaneus and the forefoot on lateral radiographs taken with the foot in dorsiflexion and plantar-flexion (Fig. 3). This is in contradistinction to other forms of convex pes plano-valgus 
with vertically disposed talus, for in these a lateral radiograph in plantar-flexion will reveal restoration of a more normal relationship of the tarsal bones (Fig. 4). This is a valuable means of differentiating the congenital vertical talus from the less resistant congenital flat foot. He also points out that, although the navicular is not demonstrable radiologically in the very young, its position can be inferred because it always lies between the ossific centre of the medial cuneiform bone and the head of the talus. The position of the navicular bone will be seen in radiographs taken in plantar-flexion to remain on the dorsum of the talus in congenital vertical talus.

TABLE I

The Point-scoring System of Clinical Evaluation

\begin{tabular}{|c|c|c|c|c|}
\hline & 4 points & 3 points & 2 points & 1 point \\
\hline Heel posture & Neutral & Trace of valgus & Moderate valgus & $\begin{array}{l}\text { Gross valgus and } \\
\text { medial malleolar } \\
\text { callosity }\end{array}$ \\
\hline Residual equinus. & $\begin{array}{c}\text { Dorsiflexion } \\
\text { above square }\end{array}$ & $\begin{array}{c}\text { Dorsiflexion } \\
\text { to square }\end{array}$ & Slight equinus & Gross equinus \\
\hline Lateral border & Convex & Straight & Slight concavity & $\begin{array}{l}\text { Gross forefoot } \\
\text { abduction }\end{array}$ \\
\hline Medial talar prominence & None & Slight prominence & $\begin{array}{l}\text { Callosity over } \\
\text { prominence }\end{array}$ & $\begin{array}{l}\text { Ulceration over } \\
\text { prominence }\end{array}$ \\
\hline Mobility . & $\begin{array}{l}\text { Useful range } \\
\text { all tarsal joints }\end{array}$ & $\begin{array}{c}\text { Subtalar joint stiff. } \\
\text { Other tarsal joints } \\
\text { mobile }\end{array}$ & $\begin{array}{l}\text { Stiffness of } \\
\text { whole tarsus }\end{array}$ & $\begin{array}{l}\text { Rigidity of } \\
\text { whole tarsus }\end{array}$ \\
\hline $\begin{array}{l}\text { Range of plantar-flexion } \\
\text { (degrees) }\end{array}$ & Over 20 & 11 to 20 & 0 to 10 & Fixed dorsiflexion \\
\hline
\end{tabular}

Stone for Lloyd-Roberts (1963) described an operation for the correction of vertical talus which consisted in a peritalar soft-tissue release, excision of the navicular bone, implantation of the tendon of tibialis anterior into the talar neck, and posterior release of the hindfoot. This has become the operation of choice for congenital vertical talus at the Hospital for Sick Children, London, and this paper presents the results in seven cases.

Initially, only those with a lengthy follow-up were to be included, but as it later became evident that the early results remained unchanged over the years and represented the final outcome, two of the more recent patients are also reported.

\section{TECHNIQUE OF OPERATION}

The patient is placed supine on the operating table and tilted towards the side to be operated upon, that leg being flexed at hip and knee and laterally rotated-the "figure 4" position.

Through a postero-medial incision a Z-lengthening of the tendo calcaneus is performed, followed by posterior capsulotomy of the ankle and subtalar joints. After identification of the neurovascular bundle, which is retracted posteriorly, the tibialis posterior tendon is traced to the navicular tuberosity and the navicular bone is excised. The medial and inferior attachments of the talus are freely divided, permitting reduction of the talus to the corrected position. The tendon of tibialis anterior is then detached distally and implanted into the talar neck by passing it vertically downwards through a hole in the bone and suturing it to itself. On two occasions the tendon of tibialis posterior has been used after rerouting it anteriorly around the medial aspect of the tibia; this practice has now been discontinued. A Kirschner wire which transfixes the talus, medial cuneiform and the first metatarsal further stabilises the reduction. 


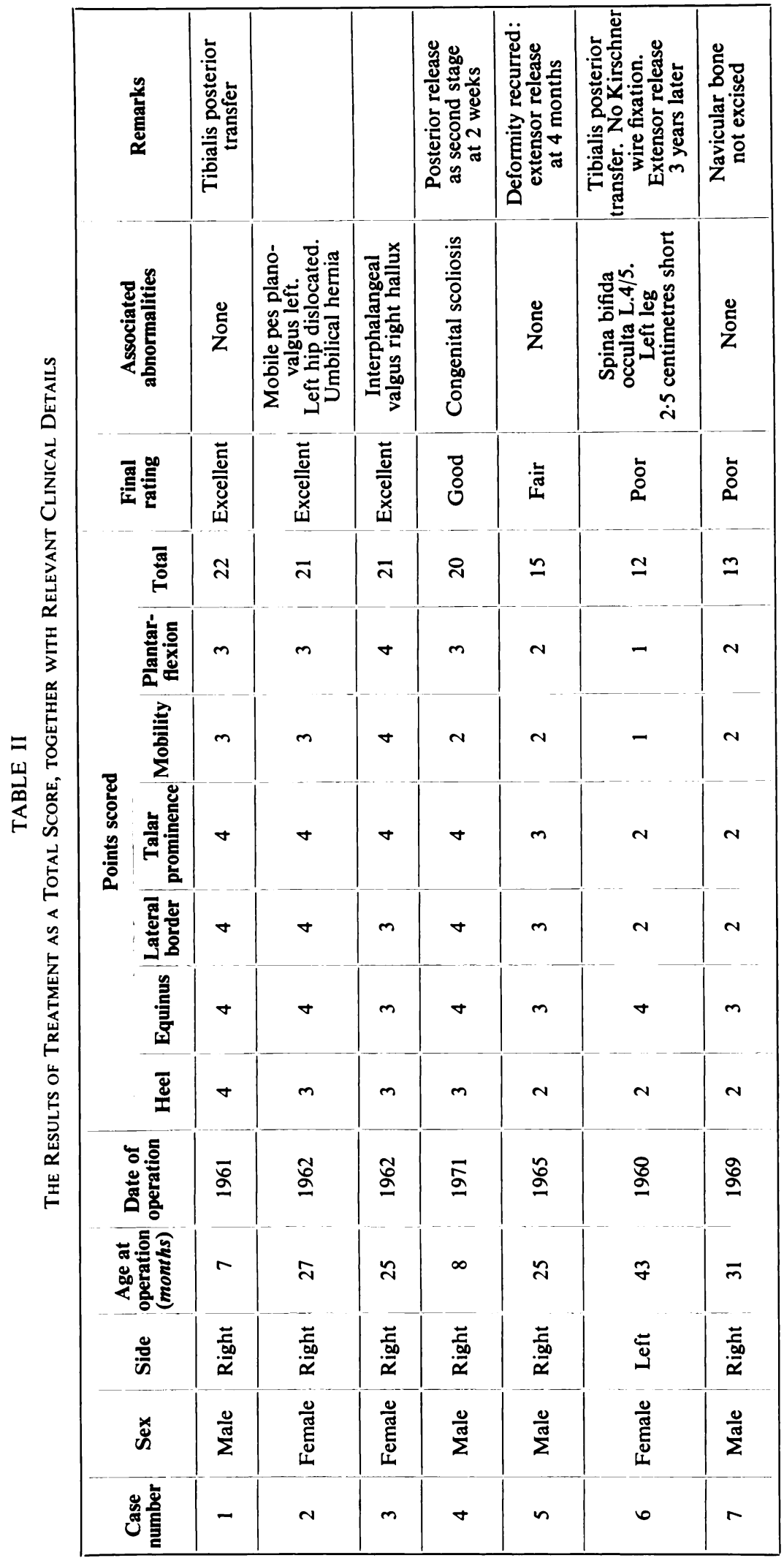

vol. 55 B, No. 3, AUGUST 1973 
After closure of the wound a knee-to-toe plaster is applied with the foot held in the corrected position. The Kirschner wire is removed three weeks after operation, and the plaster retained for a total of eight to ten weeks. Weight-bearing in the plaster is permitted from six weeks after operation. Further splinting has not been necessary.

Recently the correction has been performed in two stages, the posterior release being undertaken two to three weeks after the open reduction of the talus.
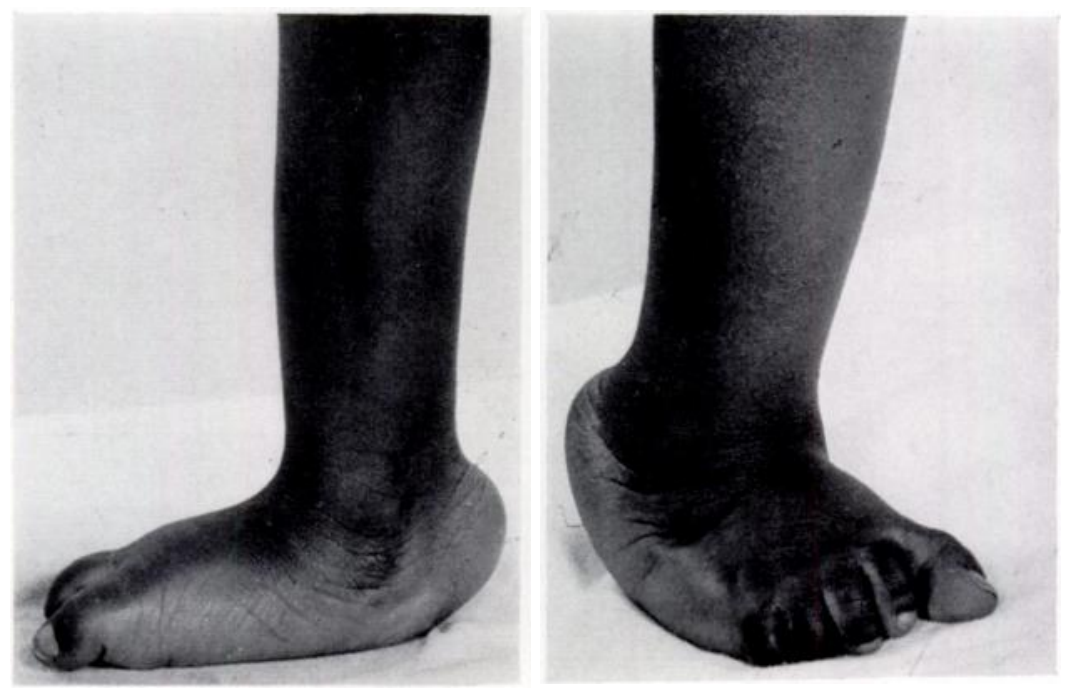

Fig. 5

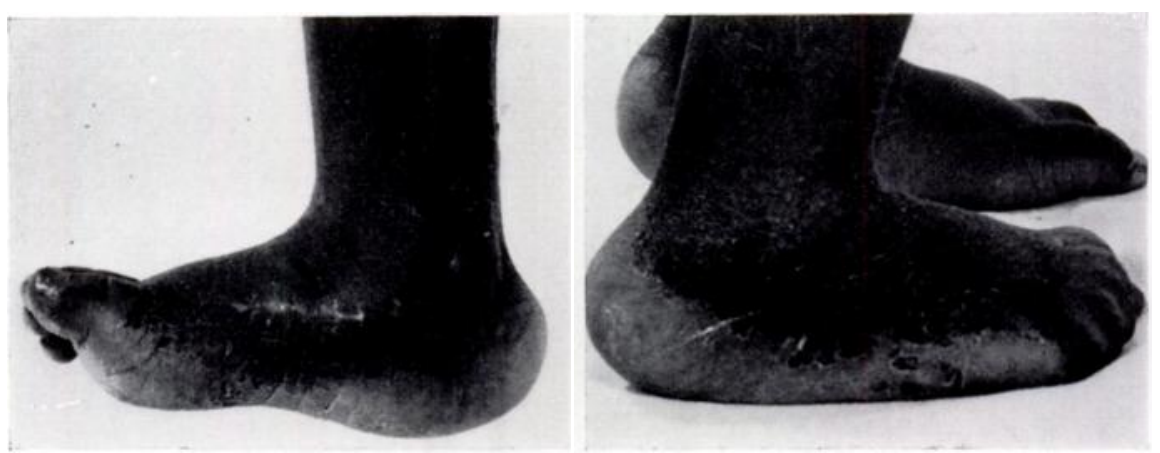

Fig. 6

A foot affected by congenital vertical talus before (Fig. 5) and four years after operation (Fig. 6).

CLINICAL MATERIAL

Seven patients are presented in whom true congenital vertical talus has been corrected by the operation described above. In one patient the navicular bone was not excised. Patients in whom the deformity was thought to be secondary to any neuromuscular disease have been excluded.

A two-stage correction was performed in one of the patients presented.

Three children exhibited congenital vertical talus as an isolated abnormality, and in the remaining four it was associated with at least one other congenital anomaly.

After operation each patient has been assessed clinically with particular attention to various features of foot shape and posture, using the point scoring system shown in Table I.

The resulting total clinical scores reflect the overall quality of the result and are classed as excellent (24-21 points), good (20-16 points), fair (15-14 points) and poor (13 points or fewer).

Antero-posterior and lateral radiographs were taken of the foot in the standing position. 


\section{RESULTS}

The clinical scores and relevant details are recorded in Table II. It can be seen that excellent or good results were achieved in four out of the seven feet. Figures 5 to 8 show the appearance of the foot before and after operation in two cases and they illustrate the degree of correction which can be achieved.

It is instructive to examine the three unsatisfactory results as there is a reason for the failure of the operation in each case.
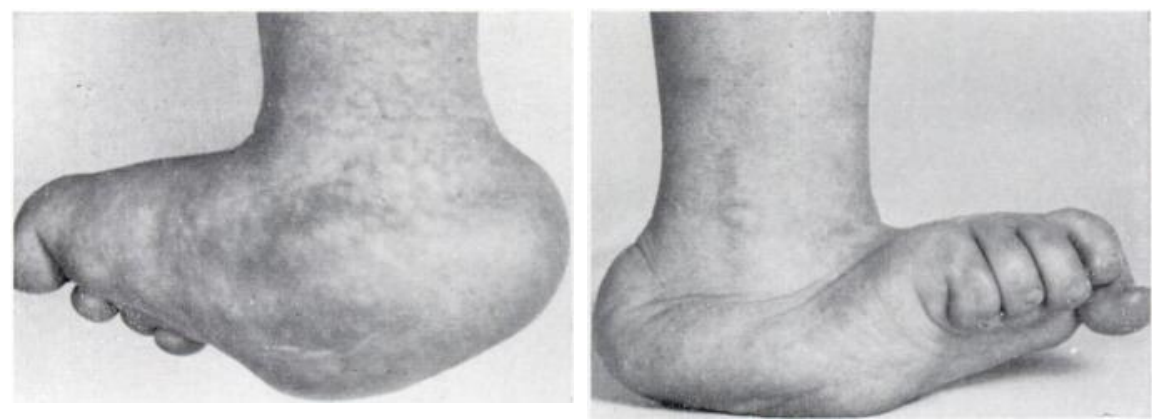

Fig. 7
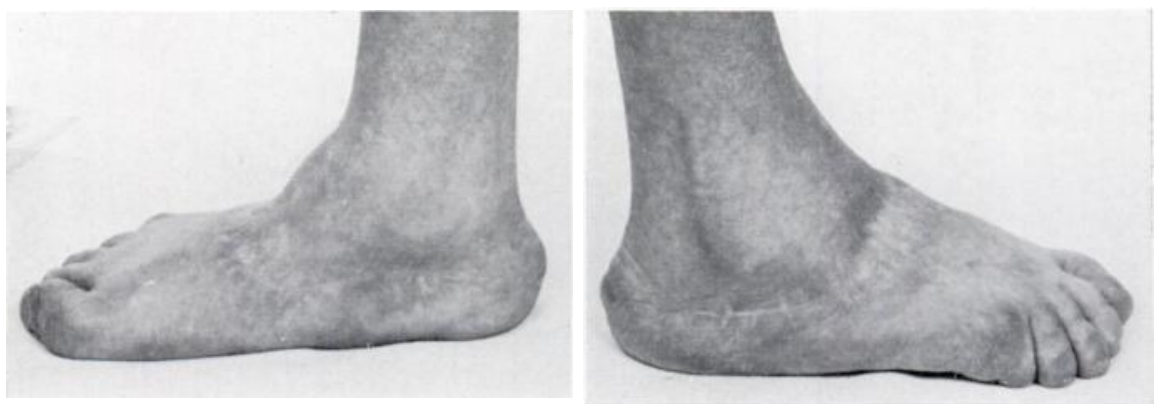

FIG. 8

Another example of correction of deformity by operation. Figure 7-Before operation. Figure 8-After operation.

In Case 7 the navicular bone was not excised because the head of the talus reduced readily after the peritalar release. Unfortunately, early relapse of the deformity occurred with medial talar prominence and a dorsiflexed, abducted forefoot. This child awaits further operation to correct this recurrent deformity.

In Case 6 a single stage release was performed exactly as described but using tibialis posterior as the motor for the tendon transfer. However, Kirschner wire fixation was omitted and the deformity started to recur soon after removal of the plaster. Further correction was attempted three years later but it has failed to result in a satisfactorily shaped foot and the child is obliged to wear surgical boots. Further features of this case were the presence of a spina bifida occulta of the fourth and fifth lumbar neural arches together with 2.5 centimetres of congenital shortening of the affected leg. This suggests the possibility of minor spinal dysraphism and, despite the absence of any detectable neurological deficit, she may be an example of the recidivous nature of neurogenic foot deformities. Radiographs of the spine were normal in all the other cases.

In Case 5, at the time of the first operation the peroneal and lateral toe extensor tendons were tight, but no release was performed. Four months later recurrent dorsiflexion and abduction of the forefoot were apparent, but despite release of the dorsal tether at that stage, the final result is disappointing. It seems that it is important to achieve full correction of all 
the deforming forces as part of the initial surgical programme. In none of the cases of congenital vertical talus was tightness of the peroneus brevis the prominent feature that it is in paralytic vertical talus.

Radiologically, the quality of the correction is reflected in the degree to which the normal talo-calcaneal relationships have been restored in both plantar-flexion and dorsiflexion of the foot. In the satisfactory corrections, the medial cuneiform articulates with the head of the talus, and no degenerative changes have as yet been seen at this joint. Slight flattening and increase in breadth of the talar head is detectable to a variable degree in the corrected cases followed up for several years (Fig. 9).

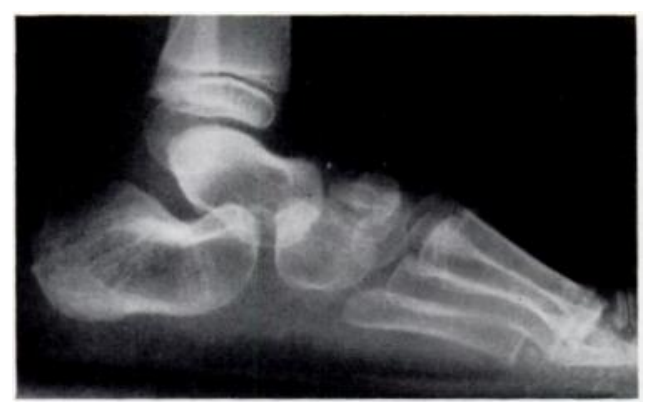

FIG. 9

Radiographs taken five years after satisfactory correction of deformity caused by congenital vertical talus, showing the talar head articulating with the medial cuneiform bone.

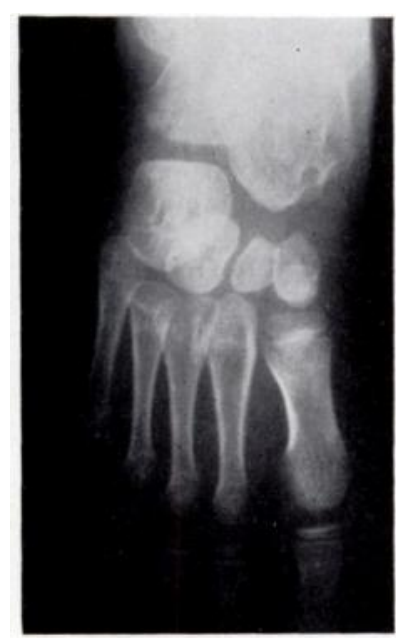

\section{DISCUSSION}

Congenital mobile pes plano-valgus is not rare and is seen in varying degrees of severity ranging from the supple, mild flat foot to the severe valgus deformity of the foot with convexity of the medial longitudinal arch. Such feet differ from those with true congenital vertical talus in that the heel is not in marked equinus, there is no rigidity, and the characteristic features of the lateral, plantar-flexion radiograph are not present. They will respond to manipulative treatment, with or without minor soft-tissue releases (Herndon and Heyman 1963. Harrold 1967, Silk and Wainwright 1967) and must be distinguished from congenital vertical talus which responds only to radical surgical correction (Hark 1950, Osmond-Clarke 1956, Lloyd-Roberts and Spence 1958, Eyre-Brook 1967). The grouping of all types of convex flat foot together has led to much confusion of terminology and to conflicting reports of the value of manipulative treatment (Lamy and Weissman 1939, Herndon and Heyman 1963, Silk and Wainwright 1967, Harrold 1967).

Congenital vertical talus is a rare condition. Osmond-Clarke (1956) reported only one out of a total attendance of 131 at a foot deformity clinic and Sonnenburg (1897) reported fifteen out of 688 patients with congenital foot deformity.

The etiology of congenital vertical talus is unknown. On the basis of a post-mortem dissection of a foot with a vertical talus deformity in a child with a thoraco-lumbar myelomeningocele, Drennan and Sharrard (1971) concluded that the underlying cause of this deformity was an "imbalance between the tibialis posterior and the evertors of the foot". Sharrard (1967) had previously incriminated weakness of the muscles supplied by the second and third sacral spinal segments as the cause. Patterson, Fitz and Smith (1968) recorded the anatomy of bilateral congenital vertical talus deformity in a six-week-old infant who had died of congenital heart disease. In their case, the tendo calcaneus was shortened and the very tight tendons of tibialis posterior and the peronei had "bow stringed" forwards of their 
respective malleoli. It was necessary to divide the tendons of tibialis anterior, peroneus brevis, extensor hallucis longus, and extensor digitorum longus before the navicular bone could be reduced on to the head of the talus. They proposed that the deformity was due to tight tendons and that all the bony changes were secondary. The navicular bone, however, had been found to be articulating with a cartilage-covered facet on the dorsum of the neck of the talus and the anterior facet of the subtalar joint was found to be absent, suggesting a primary skeletal abnormality.

There is some evidence for a genetic origin of congenital vertical talus. A number of reports list it as one of the abnormalities associated with various trisomy syndromes (Townes, DeHart, Hecht and Manning 1962; German, Rankin, Harrison, Donovan, Hogan and Bearn 1962: Uchida, Lewis, Bowman and Wang 1962). Kite (1951) stated that it might have a higher incidence in families with a predisposition to congenital talipes equinovarus and cited the case of two sisters with congenital vertical talus, one of whom had a club foot on the other side. Lamy and Weissman (1939) gave an account of congenital vertical talus in each of a pair of homozygous twins, in two brothers and also in a father and his son. Furthermore, they stressed the frequent association of congenital vertical talus with other congenital defects, and Lloyd-Roberts and Spence (1958) named arthrogryposis multiplex congenita and neurofibromatosis as concomitant abnormalities in their patients. Schinz, Baensch, Friedl and Uehlinger (1951) reported an example of congenital vertical talus in a case of Hurler's mucopolysaccharidosis.

Surgical treatment-The excellent historical review of Lamy and Weissman (1939) listed a variety of surgical procedures for the correction of congenital vertical talus but they recommended talectomy combined with a bone block to the front of the lateral malleolus. In most definitive procedures an open reduction of the talus is performed by peritalar release. and various devices have been described to retain the talus in the corrected position. Grice (1959) recommended the rerouting of tibialis anterior medial to the talar neck and its insertion into the inferior aspect of the navicular; Osmond-Clarke (1956) passed an anteriorly transposed peroneus brevis tendon through the talar neck. Kirschner wire fixation alone for a variable period after operation was advocated by Harrold (1967), and Eyre-Brook (1967) used an oblique excision of the proximal half of the navicular, placing the excised portion as a prop beneath the talar neck.

In the technique described in this paper excision of the navicular bone appears to have had no deleterious effect up to the present time and it serves to relax the medial ray of the foot so that the talus may be elevated without tension. This would be particularly the case where adaptive elongation of the talar neck has occurred. Navicular excision also reduced the tension in the extensor tendons, which must however be elongated if, after talar reduction, a dorsal tether is noted. The reduction is stabilised by implanting the tibialis anterior, hitherto a deforming force, into the talar neck and also by using a Kirschner wire for talo-cuneiform fixation. The extreme stability of reduction thus achieved is reflected in the good results, and emphasised by the disappointing outcome whenever one of the various steps has been omitted.

\section{SUMMARY}

1. Congenital vertical talus is described and the criteria for diagnosis are emphasised. The need for its distinction from other forms of convex pes valgus is stressed.

2. The operative technique of Stone for Lloyd-Roberts (1963) is detailed and the results of seven such operations are examined.

3. Departure from the technique in any respect has resulted in an unsatisfactory outcome, whereas stringent observation of the operative details has resulted in uniformly good results in four patients.

4. Certain aspects of diagnosis, differential diagnosis, etiology, pathology and surgical management are discussed, together with a review of some of the relevant literature.

vol. 55 B, No. 3, AUGUST 1973 
I would like to express my thanks, particularly to Mr G. C. Lloyd-Roberts whose great interest in this subject stimulated the present review, to the Department of Medical Photography for the preparation of the illustrations, and the Medical Records Department of the Royal National Orthopaedic Hospital for their assistance in the preparation of the typescript.

\section{REFERENCES}

Drennan, J. C., and Sharrard, W. J. W. (1971): The Pathological Anatomy of Convex Pes Valgus. Journal of Bone and Joint Surgery, 53-B, 455.

Eyre-Brook, A. L. (1967): Congenital Vertical Talus. Journal of Bone and Joint Surgery, 49-B, 618.

German, J. L., Rankin, J. K., Harrison, P. A., Donovan, D. J., Hogan, W. J., and Bearn, A. G. (1962): Autosomal Trisomy of a Group 16-18 Chromosome. Journal of Pediatrics, 60, 503.

Grice, D. S. (1959): The Role of Subtalar Fusion in the Treatment of Valgus Deformities of the Feet. American Academy of Orthopaedic Surgeons. Instructional Course Lectures, 16, 127.

HaRk, F. W. (1950): Rocker-foot Due to Congenital Subluxation of the Talus. Journal of Bone and Joint Surgery, 32-A, 344.

Harrold, A. J. (1967): Congenital Vertical Talus in Infancy. Journal of Bone and Joint Surgery, 49-B, 634.

Haveson, S. B. (1959): Congenital Flatfoot Due to Talonavicular Dislocation (Vertical Talus). Radiology, $72,19$.

Herndon, C. H., and Heyman, C. H. (1963): Problems in the Recognition and Treatment of Congenital Convex Pes Valgus. Journal of Bone and Joint Surgery, 45-A, 413.

KITE, J. H. (1951): Congenital Flatfoot. In Surgical Treatment of the Motor-Skeletal System. Second edition. Volume 1, p. 65. Edited by F. W. Bancroft and H. C. Marble. Philadelphia: J. B. Lippincott Company.

Lamy, L., and Weissman, L. (1939): Congenital Convex Pes Valgus. Journal of Bone and Joint Surgery, $21,79$.

Lloyd-Roberts, G. C., and Spence, A. J. (1958): Congenital Vertical Talus. Journal of Bone and Joint Surgery, 40-B, 33.

Osmond-Clarke, H. (1956): Congenital Vertical Talus. Journal of Bone and Joint Surgery, 38-B, 334.

Patterson, W. R., Fitz, D. A., and Smrth, W. S. (1968): The Pathologic Anatomy of Congenital Convex Pes Valgus. Journal of Bone and Joint Surgery, 50-A, 458.

Schinz, H. R., Baensch, W. E., Friedl, E., and Uehlinger, E. (1951): Roentgen-Diagnostics. Volume 1, p. 733. Skeleton, Part 1. Translated by J. T. Case. London: William Heinemann Ltd.

Sharrard, W. J. W. (1967): Paralytic Deformity in the Lower Limb. Journal of Bone and Joint Surgery, 49-B, 731.

SiLK, F. F., and WAINwright, D. (1967): The Recognition and Treatment of Congenital Flat Foot in Infancy. Journal of Bone and Joint Surgery, 49-B, 628.

SONNENBURG, S. (1897): Klumpfuss. In Real-Encyclopädie der gesammten Heilkunde. Medicinisch-chirurgisches Handwörterbuch für praktische Aerzte. Third edition. Volume 12, p. 368. Edited by A. Eulenburg. Wien: Urban and Schwarzenberg.

Stone, K. H. (for Lloyd-Roberts, G. C.) (1963): Congenital Vertical Talus: A New Operation. Proceedings of the Royal Society of Medicine, 56, 12.

Townes, P. L., DeHart, G. K., Jun., HeCht, F., and Manning, J. A. (1962): Trisomy 13-15 in a Male Infant. Journal of Pediatrics, 60, 528.

Uchida, I. A., Lewis, A. J., Bowman, J. M., and Wang, H. C. (1962): A Case of Double Trisomy: Trisomy No. 18 and Triplo-X. Journal of Pediatrics, 60, 498. 